\title{
Endoscopic Detection of Gastric Carcinoma After Helicobacter pylori Eradication: More Difficult or Just Hype?
}

\author{
Toshihisa Takeuchi $^{1} \cdot$ Kazuhide Higuchi $^{1}$
}

Received: 26 February 2016/ Accepted: 28 February 2016/Published online: 17 March 2016

(c) Springer Science+Business Media New York 2016

Since Helicobacter pylori $(\mathrm{Hp})$ infection is thought to be an essential prerequisite for the development of gastric carcinoma (GC), occurrence without Hp infection is uncommon. Since Hp eradication inhibits cancer development, and since eradication therapy is now universal, consideration must therefore be given to distinguishing post-eradication patients from uninfected patients. At present, there are few guidelines that inform GC surveillance in posteradication patients, adding to the importance of being aware of the endoscopic characteristics of the type of GC that develops after eradication.

Including the reports by Yamamoto et al. [1] and Matsuo et al. [2], $>10$ studies of GC detected after Hp eradication have been published to date [3-10]. The characteristics of post-eradication GC include: (a) detection $\sim 40$ months after eradication; (b) lesions are histologically well differentiated; (c) gross lesion morphology is depressed; and (d) the lesions are small (10-20 mm diameter). Pathologically, the Ki-67 index and Wnt5a expression are significantly reduced [1, 11]. In some patients, the mucosa surrounding the lesion is obscured [11], possibly due to the flattening of the tumor tissue, with formation of non-neoplastic foveolar epithelium as the outermost layer.

In this issue of Digestive Diseases and Sciences, Hori et al. report a retrospective analysis of GC for which endoscopic submucosal dissection was carried out, comparing the characteristics of 59 lesions after $\mathrm{Hp}$ eradication

Kazuhide Higuchi

higuchi@osaka-med.ac.jp

1 Second Department of Internal Medicine, Osaka Medical College, 2-7 Daigaku-machi, Takatsuki, Osaka 569-8686, Japan (the post-eradication group) and 152 lesions positive for $\mathrm{Hp}$ (the infected group) [12]. The findings in the post-eradication group were mostly similar to those reported previously in that the median time to detection of the GC was 39 months, the lesion diameter was $\sim 10 \mathrm{~mm}$, and the gross morphology was of the depressed type. Moreover, lesions in the post-eradication were significantly more covered with non-tumor epithelium than were the GC in the Hp-positive group. Infiltration was also increased in the post-eradication group, suggesting that cancer proliferation kinetics had changed after Hp eradication. Furthermore, eradication reduced histological inflammation in the surrounding mucosa, the cancer tissue generally had a whitish appearance, and the inhibition of the inflammatory response to the cancer might have flattened the lesion.

Recent advances in the immunohistochemical identification of mucin phenotypes have improved the understanding of GC development. Although intestinal-type differentiated cancers are usually associated with atrophy and intestinal metaplasia, differentiated cancers of the gastric phenotype also exist; indeed, in most cases of GC, maintenance of the gastric mucin phenotype is preserved during the initial onset. Then, as the tumor grows, the intestinal phenotype is acquired close to the proliferative zone, finally resulting in the preponderance of the intestinal phenotype, a process attributed to Hp infection [13]. Hp eradication inhibits formation of intestinal-type cancer, allowing emergence of the gastric type, an additional cause of the difference in morphology between GC with $\mathrm{Hp}$ infection and after $\mathrm{Hp}$ eradication. Using magnifying endoscopy with narrow-band imaging (NBI) of GC, Kobayashi et al. reported that $\sim 40 \%$ of differentiatedtype cancers had evidence of gastritis, blurring differentiation of cancer from normal tissues [14]. For post-eradication GC, the lesions are likely to be difficult to detect 
endoscopically, as they are small, flattened, or depressed in shape, with whitish discoloration. Full recognition of these characteristics has the potential to contribute to early detection of GC.

\section{References}

1. Yamamoto K, Kato M, Takahashi M, et al. Clinicopathological analysis of early-stage gastric cancers detected after successful eradication of Helicobacter pylori. Helicobacter. 2011;16:210-216.

2. Matsuo T, Ito M, Tatsugami M, et al. Gastric Cancer development after Helicobacter pylori eradication therapy: a new form of gastric neoplasia. Digestion. 2012;85:61-67.

3. Kato M, Asaka M, Ono S, et al. Eradication of Helicobacter pylori for primary gastric cancer and secondary gastric cancer after endoscopic mucosal resection. J Gastroenterol. 2007;42:16-20.

4. Takenaka R, Okada H, Kato J, et al. Helicobacter pylori eradication reduced the incidence of gastric cancer, especially of the intestinal type. Aliment Pharmacol Ther. 2007;25:805-812.

5. Ogura T, Hirata Y, Yanai A, et al. The effect of Helicobacter pylori eradication on reducing the incidence of gastric cancer. $J$ Clin Gastroenterol. 2008;42:279-283.

6. Yanaoka K, Oka M, Ohata H, et al. Eradication of Helicobacter pylori prevents cancer development in subjects with mild gastric atrophy identified by serum pepsinogen levels. Int J Cancer. 2009;125:2697-2703.
7. Mabe K, Takahashi M, Oizumi H, et al. Does Helicobacter pylori eradication therapy for peptic ulcer prevent gastric cancer? Word J Gastroenterol. 2009;15:4290-4297.

8. Take S, Mizuno M, Ishiki K, et al. The long-term risk of gastric cancer after the successful eradication of Helicobacter pylori. $J$ Gastroenterol. 2011;46:318-324.

9. Kamada T, Hata J, Sugiu K, et al. Clinical features of gastric cancer discovered after successful eradication of Helicobacter pylori: results from a 9-years prospective follow-up study in Japan. Aliment Pharmacol Ther. 2005;21:203-206.

10. Tashiro J, Miwa J, Tomita T, et al. Gastric cancer detected after Helicobacter pylori eradication. Dig Endosc. 2007;19:167-173.

11. Ito $M$, Tanaka $S$, Takata $S$, et al. Morphological changes in human gastric tumors after eradication therapy of Helicobacter pylori in a short-term follow up. Aliment Pharmacol Ther. 2005;21:559-566.

12. Hori K, Watari J, Yamasaki Y, et al. Morphological characteristics of early gastric neoplasms detected after Helicobacter pylori eradication. Dig Dis Sci. (Epub ahead of print). doi:10. 1007/s10620-015-3887-2.

13. Kakinoki R, Kushima R, Matsubara A, et al. Reevaluation of histogenesis of gastric carcinoma: a comparative histopathological study between Helicobacter pylori-negative and $\mathrm{H}$. pyloripositive cases. Dig Dis Sci. 2009;54:614-620.

14. Kobayashi M, Hashimoto S, Nishikura K, et al. Magnifying narrow-band imaging of surface maturation in early differentiated-type gastric cancers after Helicobacter pylori eradication. $J$ Gastoroenterol. 2013;48:1332-1342. 